\title{
Contribution à l'identification des larves de Chironomidae du fleuve Niger à Niamey
}

\author{
Bassirou ALHOU ${ }^{1 *}$ et Boudewijn GODDEERIS ${ }^{2}$ \\ ${ }^{1}$ University Abdou Moumouni of Niamey, Faculty of sciences, Department of biology, B.P: 10,662 Niamey \\ (Niger),E-mail:alhoubass@yahoo.fr \\ ${ }^{2}$ Royal Belgian Institute of Natural Sciences, RBINS, Freshwater Biology, Rue Vautier 29, B-1000 Brussels, \\ E-mail: Boudewijn.Goddeeris@natuurwetenschappen.be \\ *Auteur correspondant ; E-mail: alhoubass@yahoo.fr; Tel: (00227) 96961422; fax: (00227) 20733072)
}

\section{RESUME}

Les Chironomidae sont des bons indicateurs de la pollution des eaux du fleuve Niger à Niamey. Dans la perspective de leur utilisation dans le suivi régulier d'éventuelles altérations de la qualité des eaux du fleuve, il est indispensable de disposer d'un outil simple d'identification des taxons facilement utilisable par les agents de terrain. Cette description qui présente de manière illustrée les caractéristiques morphologiques de vingt taxons (Ablabesmyia sp., Cf. Procladius sp., Clinotanypus sp., Micropelopiinae sp., Orthocladiinae sp., Chironomini sp.1, Chironomini sp.2, Chironomus gr. Plumosus, Cryptochironomini sp., Cryptochironomus sp. 1, Cryptochironomus sp. 2, Dicrotendipes sp., Glyptotendipes sp., Microchironomus sp., Nilodosis sp., Parachironomus sp., Polypedilum spp. 1, Polypedilum spp.2, Xenochirnomus sp., Tanytarsini spp.) récoltés le long du fleuve Niger à Niamey s'inscrit dans ce cadre. Ces taxons sont répartis en trois sous familles à savoir les Tanypodinae, les Orthocladiinae et les Chironominae. La description des taxons s'appuie sur les caractéristiques suivantes : la présence des branchies, des soies, la forme de la capsule céphalique, les yeux (forme, position, nombre de paires), le mentum, les dents mandibulaires, la forme de la plaque paralabiale et la structure des antennes.

(C) 2010 International Formulae Group. All rights reserved.

Mots clés : Chironomidae, fleuve Niger, qualité de l'eau, Niamey, Tanypodinae, Orthocladiinae, Chironominae.

\section{INTRODUCTION}

Les Chironomidae constituent une famille de diptère suscitant beaucoup d'intérêts tant par sa diversité que par son intérêt écologique notamment dans l'étude des changements climatiques et environnementaux, et de la qualité des eaux des lacs et des rivières (Callisto et al., 2002 ; Alhou, 2008). Les connaissances taxonomique et écologique de cette famille des diptères restent encore fragmentaires (Eggermont et
Verschuren, 2003). Sa taxonomie évolue assez rapidement avec de nouvelles subdivisions liées à l'apparition au fil du temps de nouvelles espèces (Vardal et al., 2002). En Afrique de l'Ouest, contrairement à l'Afrique centrale et orientale, il existe très peu de données sur la taxonomie des larves de Chironomidae (Diomandé et al., 2000).

L'intérêt que suscitent les chironomides dans l'étude de la qualité des eaux du fleuve Niger à Niamey (Alhou, 2008) nécessite la 
mise au point d'une diagnose directement utilisable par les agents de terrain afin de faire un premier travail d'identification des taxons.

Cette diagnose représente un outil pratique pouvant servir à un premier inventaire de la biodiversité chironomidienne du fleuve Niger à Niamey en l'absence d'une clé appropriée pour l'identification des Chironomidae de l'Afrique de l'Ouest.

Elle est essentiellement élaborée à partir des échantillons provenant du fleuve Niger à Niamey en se référant à la littérature déjà existante (Eggermont et Verschuren, 2003, 2004a, 2004b ; Eggermont, 2004 ; Eggermont et al., 2005).

Cette étude a pour objectif de contribuer à la connaissance des Chironomidae du fleuve Niger et à la mise en place d'un outil de terrain pour l'inventaire de la biodiversité chironomidienne.

\section{MATERIEL ET METHODES}

Zone d'étude

L'étude est menée le long du fleuve Niger de l'amont à l'aval de la ville de Niamey (Alhou, 2008 et 2009). Cette dernière se situe dans la partie occidentale du Niger entre $13^{\circ} 31^{\prime}$ de latitude Nord et $2^{\circ} 26^{\prime}$ de longitude Est. Elle se situe à une altitude de $218 \mathrm{~m}$ (Fig. 1).

Niamey appartient à un climat sahélien caractéristique des régions semi-arides avec une alternance dans l'année d'une saison des pluies (juin, juillet, août) et d'une saison sèche (septembre à mai).

La moyenne pluviométrique annuelle de 1995 à 2005 est de $518 \mathrm{~mm}$ avec un minimum de $386 \mathrm{~mm}$ en 2002 et un maximum exceptionnel de $817 \mathrm{~mm}$ en 1998. L'écart type est de $123 \mathrm{~mm}$. Elle présente un histogramme unimodal. Le mois le plus pluvieux de l'année est le mois d'août avec 173 mm en moyenne.

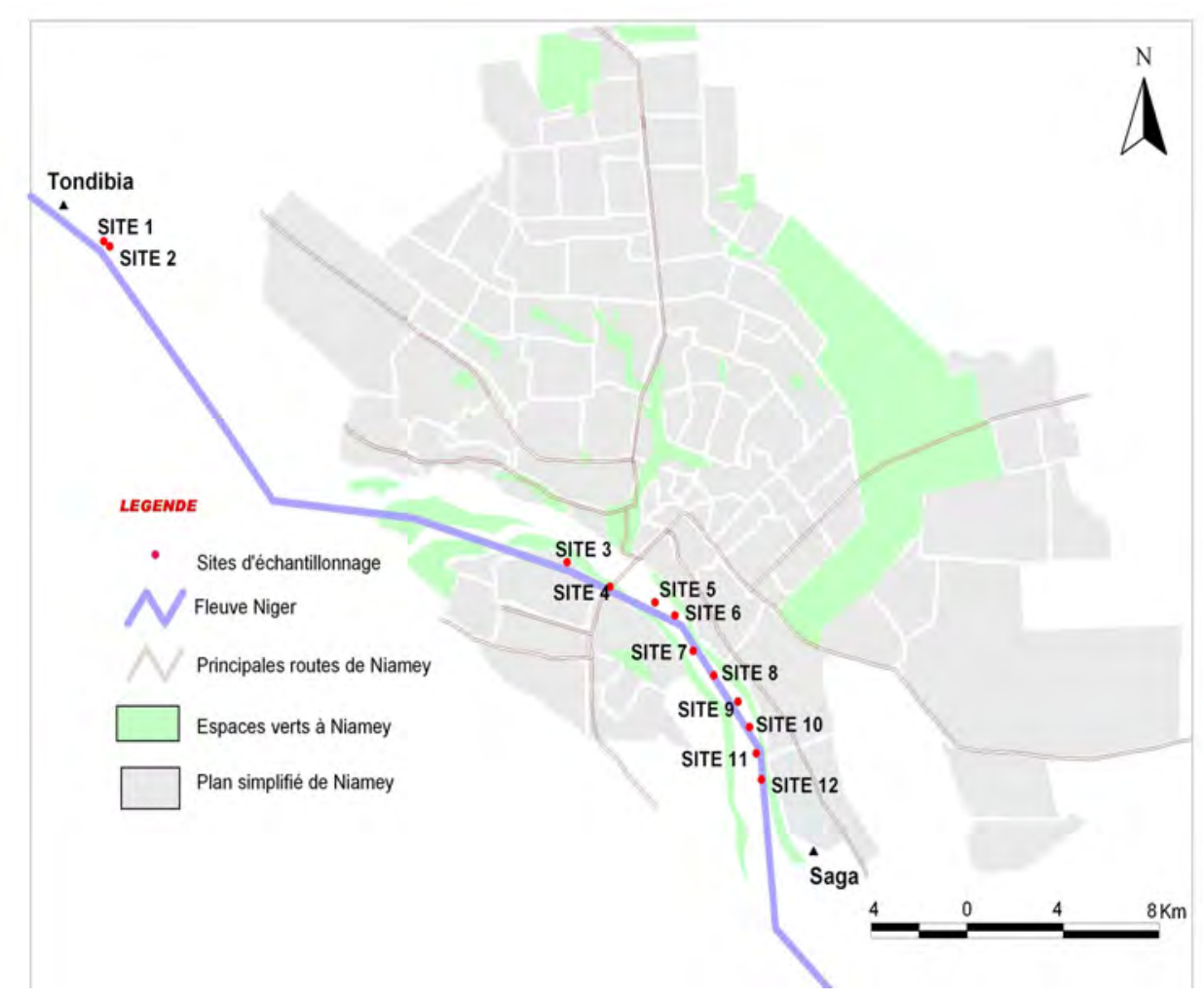

Figure 1 : Localisation des sites d'échantillonnage de l'amont (site 1 et 2) à l'aval (site 12) de la ville de Niamey. 
La température moyenne annuelle de 1995 à 2004 est de $30{ }^{\circ} \mathrm{C}$. Les températures les plus élevées sont enregistrées aux mois d'avril et de mai avec $35{ }^{\circ} \mathrm{C}$. Les mois de décembre $\left(26^{\circ} \mathrm{C}\right)$ et janvier $\left(25^{\circ} \mathrm{C}\right)$ sont les plus frais. L'écart type est de $3{ }^{\circ} \mathrm{C}$.

La période humide de l'année va de juillet à septembre avec un taux supérieur à $60 \%$.

Les échantillons sont prélevés en aval immédiat des points de rejet, mais aussi en amont et en aval lointain de ces points de rejet.

\section{Préparation et identification des taxons}

Une fois récoltées, les larves de Chironomidae sont triées de manière minutieuse avant de procéder à une première détermination des taxons, d'abord sans préparation, puis ensuite on procède à la préparation des spécimens non immédiatement identifiés (Alhou, 2008).

L'identification des taxons est basée sur la présence des branchies, des soies, la forme de la capsule céphalique, les yeux (forme, position, nombre de paires), le mentum, les dents mandibulaires, la forme de la plaque paralabiale et la structure des antennes (Eggermont et Verschuren, 2003, 2004a, 2004b ; Eggermont, 2004; Eggermont et al., 2005 ; Alhou, 2008).

Cette diagnose met l'accent sur les caractéristiques morphologiques ci-dessus mentionnées et son utilisation est facilitée par des illustrations fournies à partir des prises de vue. Elle ne donne pas une description détaillée des taxons mais se limite aux caractéristiques principales qui permettent une détermination des taxons au niveau générique.

\section{RESULTATS}

Trois sous-familles de la famille des Chironomidae ont été récoltées le long du fleuve Niger à Niamey. Il s'agit de :

- Tanypodinae : Une tache oculaire plus ou moins réniforme de chaque côté de la tête ; tête toujours plus ou moins allongée et (sub-) triangulaire vue de côté ; tubercules préanaux fort développés et allongés; labium sans plaques paralabiales.

- Orthocladiinae: Deux taches oculaires de chaque côté de la tête, l'inférieure placée plus en avant que la supérieure; tête assez trapue, rarement très allongée ; tubercules préanaux peu développés ou assez courts; labium avec plaques paralabiales non striées.

- Chironominae : Deux taches oculaires de chaque côté de la tête, l'un en dessous de l'autre; tête trapue, rarement allongée ; tubercules préanaux peu développés ou assez courts; labium avec plaques paralabiales striées.

Les diagnoses qui suivent sont élaborées d'après les larves du quatrième stade (= dernier stade larvaire). Les larves du troisième stade peuvent être identifiées avec ces diagnoses en tenant compte que les proportions des segments des antennes sont différentes.

Les différentes espèces sont ci-dessous décrites :

\section{Sous-famille des Tanypodinae Ablabesmyia sp.}

Une larve appartenant à la sous-famille des Tanypodinae avec les caractéristiques suivantes (Fig. 2) : Capsule céphalique allongée, 2 fois aussi long que large ; les bords latéraux, par vue dorsale, un peu anguleux postérieurement; le corps non particulièrement «poilu»; deux crochets bruns au pseudopode postérieur.

\section{Cf. Procladius $s p$.}

Une larve appartenant à la sous-famille des Tanypodinae avec les caractéristiques suivantes (Fig. 3) : Capsule céphalique trapue, c'est-à-dire longueur moins que 1,5 fois la largeur. Glossa à cinq dents noirâtres avec le bord antérieur concave; franges latérales de soies aux segments abdominaux 1 à 7 (chez Procladius 1 à 6).

\section{Clinotanypus sp.}

Une larve appartenant à la sous-famille des Tanypodinae avec les caractéristiques 
suivantes (Fig. 4): Capsule céphalique allongée, c'est-à-dire longueur \pm 2 fois la largeur; antennes longues, c'est-à-dire longueur $\pm 2 / 3$ de la longueur de la tête ; franges latérales de soies aux segments abdominaux 1 à 7 .

\section{Micropelopiinae sp.}

Une larve appartenant à la sous-famille des Tanypodinae avec les caractéristiques suivantes (Fig. 5) : Capsule céphalique allongée, 2 fois aussi longue que large ; tout le corps «poilu» de longues soies, plus longues que le diamètre du segment et bien visibles (par exemple 10 sur le prothorax), mais pas de franges latérales de soies; plusieurs crochets brunâtres à chaque pseudopode postérieur ; glossa à cinq dents noirâtres avec le bord antérieur concave.

\section{Sous-famille des Orthocladiinae Orthocladiinae sp.}

Une larve appartenant à la sous-famille des Orthocladiinae avec les caractéristiques suivantes (Fig. 6) : Forme du labium triangulaire, avec une dent médiane large (largeur des latérales 1 à 3 réunies) et arrondie, et six dents latérales sub-égales, pointues vers l'intérieur; plaque paralabiale non-développée; mandibule avec une dent terminale bien développée, pointue et noirâtre et quatre dents intérieures pointues et noirâtres, dont la quatrième est plus importante que les autres, mais fusionnée avec le corps de la mandibule.

\section{Sous-famille des Chironominae Chironomini sp.1}

Une larve appartenant à la sous-famille des Chironominae avec les caractéristiques suivantes (Fig. 7): Antennes à six segments (proportion de la longueur des segments 1 à 6 = 8-3-4-2-4-1); un organe de Lauterborn au bout du segment 2 et un au bout du segment 3) ; labium fort coloré ; la dent médiane trilobée (la première latérale complètement fusionnée avec la médiane) ; la taille des dents latérales 2 à 7 diminue progressivement.

\section{Chironomini sp.2}

Une larve appartenant à la sous-famille des Chironominae avec les caractéristiques suivantes (Fig. 8): Antennes très caractéristiques et uniques pour un chironome, c'est-à-dire à quatre segments, avec un organe de Lauterborn dans la partie inférieure, à un tiers de la hauteur du segment 2 et un subterminal sur le même segment (le segment 3 originel, extrêmement petit, semble fusionné avec le segment 2 ; labium avec deux dents médianes et six dents latérales; toutes les dents pointues et diminuant progressivement en taille vers l'extérieur.

\section{Chironomus gr. plumosus}

Une larve appartenant à la sous-famille des Chironominae avec les caractéristiques suivantes (Fig. 9) : Deux paires de branchies ventrales très allongées au huitième segment abdominal; une paire de branchies latérales au bout du septième segment.

\section{Cryptochironomini sp.}

Une larve appartenant à la sous-famille des Chironominae avec les caractéristiques suivantes (Fig. 10) : Labium plus ou moins rectiligne, la partie médiane peu saillante ; la dent médiane large et claire; la première dent latérale, foncée, collée contre la médiane ; la dent médiane avec les deux latérales 1 , très large, c'est-à-dire plus large que les dents latérales 2 à 4 réunies (d'un côté); plaques paralabiales larges et s'amincissant vers extérieur; les dents latérales 2 à 4 carrées et sub-égales; les dents latérales 5 à 7 formant un groupe dont la 6 est grande, saillante et pointue ; 5 et 7 à peine visibles; antenne avec cinq segments; le premier segment plus long que le flagellum (segments 2 à 5 réunis); segment 2 large et trapu; chète implanté au milieu du segment 2. Segment 3 svelte et aussi long que le 2 ; segments 4 et 5 minuscules.

\section{Cryptochironomus sp. 1}

Une larve appartenant à la sous-famille des Chironominae avec les caractéristiques 
suivantes (Fig. 11) : Labium concave ; la dent médiane claire; la première dent latérale, foncée, collée contre la médiane; la dent médiane avec les deux latérales 1, relativement étroite, c'est-à-dire plus ou moins la largeur des dents latérales 2 à 4 réunies (d'un côté); plaques paralabiales larges et s'amincissant vers l'extérieur; les dents latérales 2 à 7 pointues; les dents 2 à 4 sub-égales, la taille des dents 4 à 7 diminuant progressivement: la ligne antérieure formée par la pointe des dents latérales 2 à 7 légèrement convexe; antennes avec cinq segments; le premier segment un peu plus court que la longueur du flagellum (segments 2 à 5 réunis); segment 3 un rien plus long que le 2 ; segments 4 et 5 minuscules; chète de l'antenne inséré sur le côté distale du segment 2.

\section{Cryptochironomus sp. 2}

Une larve appartenant à la sous-famille des Chironominae avec les caractéristiques suivantes (Fig. 12) : Labium concave ; la dent médiane claire; la première dent latérale, foncée, collée contre la médiane; la dent médiane avec les deux latérales 1, relativement large, c'est-à-dire plus large que les dents latérales 2 à 4 réunies (d'un côté) ; plaques paralabiales larges et s'amincissant vers le côté extérieur ; les dents latérales 2 à 4 pointues ; les dents 2 et 3 sub-égales, les 4 à 7 diminuant progressivement; la pointe des dents 5 à 7 tronquée ; les dents 6 et 7 plus ou moins fusionnées à leur base; la ligne antérieure formée par la pointe des dents latérales 2 à 7 presque rectiligne; antennes avec cinq segments ; le premier segment aussi long que le flagellum (segments 2 à 5 réunis) ; segment 3 un rien plus long que le 2 ; segments 4 et 5 minuscules; chète de l'antenne inséré sur le côté distale du segment 2.

\section{Dicrotendipes $s p$.}

Une larve appartenant à la sous-famille des Chironominae avec les caractéristiques suivantes (Fig. 13) : La plaque frontale (frontal apotome) à bord antérieur creux et avec des bourrelets; les dents du labium pointues et colorées ; la dent médiane simple ; six dents latérales de chaque côté ; la latérale 1 aussi haute que la médiane; la latérale 2 beaucoup plus petite et fusionnée à la base de la latérale 1 ; les 3 à 6 plus petites que la 1 et diminuant en taille vers l'extérieur; plaque paralabiale assez étroite, fort striée et à bord antérieur crénelé.

\section{Glyptotendipes sp.}

Une larve appartenant à la sous-famille des Chironominae avec les caractéristiques suivantes (Fig. 14) : Tête forte et trapue, assez fort colorée; insertion de la cuticule sur la capsule céphalique bien devant le bord occipitale (une bande postérieure de la capsule céphalique est interne); la plaque frontale (frontal apotome) à bord antérieur creux ; une paire de branchies ventrales à la partie postérieure du huitième segment abdominal.

\section{Microchironomus sp.}

Une larve appartenant à la sous-famille des Chironominae avec les caractéristiques suivantes (Fig. 15) : Labium coloré, avec une dent médiane trilobée et pointue (les trois lobes sont pointus); les six dents latérales pointues; la quatrième et la sixième beaucoup plus petites que la cinquième, qui a la même taille que la troisième ; les deux dents latérales de la mandibule non-saillantes et plutôt carrées; la petite taille de la larve, dont le corps s'amincit vers l'arrière et le thorax verdâtre sont des caractéristiques qui facilitent l'identification.

\section{Nilodosis sp.}

Une larve appartenant à la sous-famille des Chironominae avec les caractéristiques suivantes (Fig. 16) : Tête brunâtre et allongée ; la partie antérieure, au niveau du labre, tronquée et anguleuse vue de dessus ; vue de côté, en forme de coin et s'amincissant vers l'avant ; labium concave, c'est-à-dire la partie médiane plus profonde que les deux côtés latéraux, dont le bout des dents forment une courbe convexe; la double dent médiane un peu plus claire que les sept dents latérales; la 
latérale 4 est la plus grande et la taille des latérales diminue progressivement vers l'intérieur et l'extérieur à partir de la 4 ; tubercules préanaux développés et plus longs que larges; papilles anales longues et étranglées au milieu; les deux dernières caractéristiques ont été utilisées pour faciliter la séparation des espèces.

\section{Parachironomus sp.}

Une larve appartenant à la sous-famille des Chironominae avec les caractéristiques suivantes (Fig. 17) : Capsule céphalique claire, non sclérosée; la tache oculaire inférieure dédoublée, c'est-à-dire une petite tache oculaire supplémentaire au-dessus et contre le devant de la tache oculaire inférieure ; plaque paralabiale assez étroite ; le bord antérieur denté de quelques pointes aiguës ; lobe extérieur de la base de la plaque fort développé vers l'arrière et avec des stries recourbées vers l'avant; labium clair; la dent médiane simple et pointue ; 7 dents latérales pointues.

\section{Polypedilum spp. 1}

Une larve appartenant à la sous-famille des Chironominae avec les caractéristiques suivantes (Fig. 18) : Antenne à 5 segments ; labium à deux dents médianes et à 7 dents latérales, toutes pointues et formant une rangée un peu courbée ; la latérale 2 aussi haut que la médiane; la latérale 1 petite, n'atteignant que la mi-hauteur de la latérale 2 ; les latérales 3 à 7 diminuant progressivement en taille vers l'extérieur.

\section{Polypedilum spp. 2}

Une larve appartenant à la sous-famille des Chironominae avec les caractéristiques suivantes (Fig. 19) : Antenne à 5 segments ; labium à deux dents médianes et à 7 dents latérales, toutes pointues et formant une rangée un peu courbée ; la latérale 2 aussi haut que la médiane ; la latérale 1 un rien plus petit que la médiane; les latérales 3 à 7 diminuant progressivement en taille vers l'extérieur.

\section{Xenochirnomus sp.}

Une larve appartenant à la sous-famille des Chironominae avec les caractéristiques suivantes (Fig. 20): Tête assez étroite, surtout vers l'avant; la tache oculaire inférieure plus grande et assez près de la supérieure ; touffe de lamelles sur le labre (seulement visible quand le labre est étendu); labium très typique et unique : une dent médiane simple, bien développée mais placée dans une dépression dans laquelle se trouve également la latérale 1 , très petite et orientée vers la latérale 2 ; la latérale 2 un peu plus étroite que la dent médiane, mais placée plus haut et dépassant toutes les autres dents; la latérale 3 petite et collée contre la 2 ; les latérales 4 à 8 assez petites et diminuent en taille vers l'extérieur.

\section{Tanytarsini spp.}

Une larve appartenant à la sous-famille des Chironominae avec les caractéristiques suivantes (Fig. 21): Antenne sur un socle bien développé, plus long que large; plaques paralabiales basses et très larges; plusieurs genres sont présents dans les échantillons.

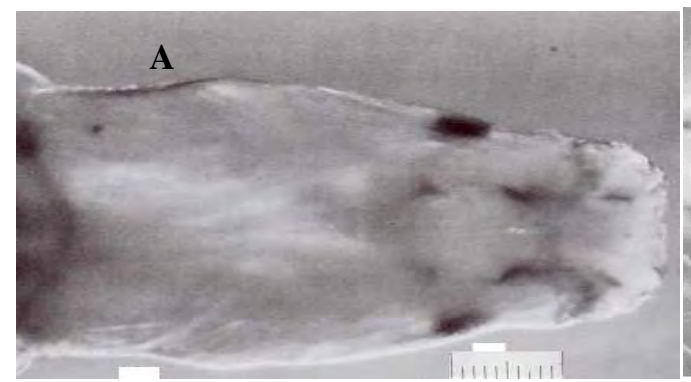

$80 \times 10$

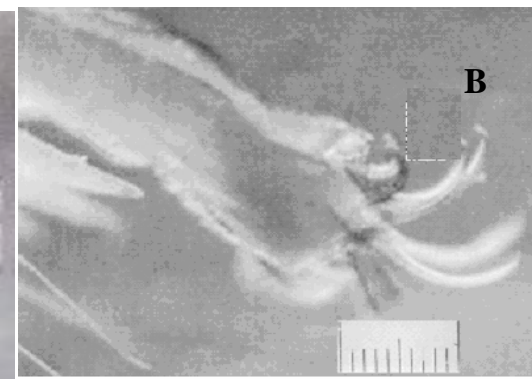

$80 \times 10$

Figure 2 : Ablabesmyia sp. (A= capsule céphalique anguleuse ; $\mathrm{B}=$ pseudopode postérieur ; échelle sur la photo $=1 \mathrm{~mm}$ ). 


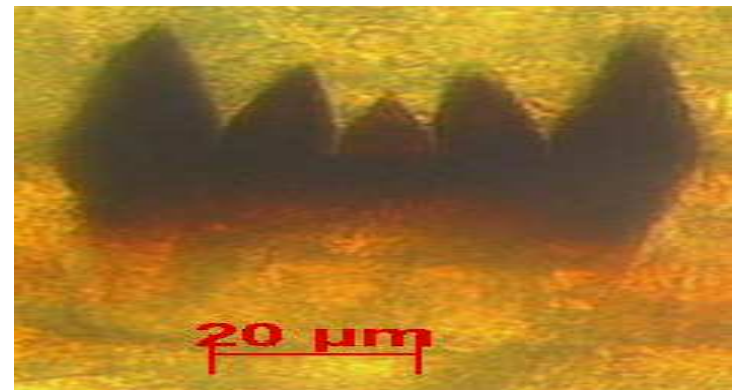

Figure 3 : Cf. Procladius sp. (Mentum)

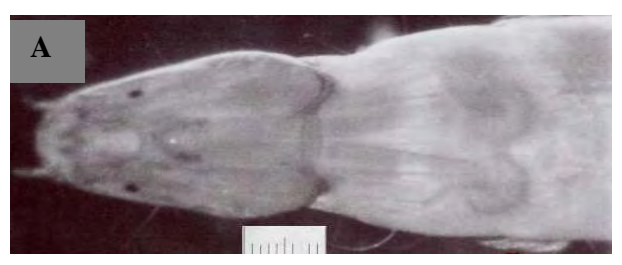

$80 \times 10$

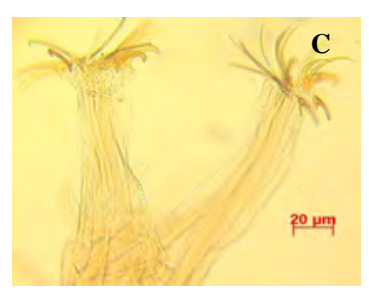

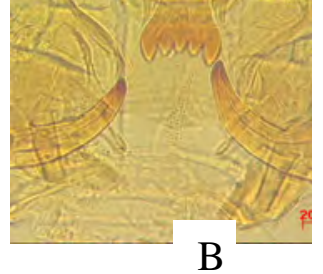

B

Figure 4 : Clinotanypus sp. (A = Capsule céphalique plus franges de soies sur le corps, $\mathrm{B}=$ mentum, $\mathrm{C}=$ pseudopodes postérieurs, échelle sur la photo $=1 \mathrm{~mm})$.
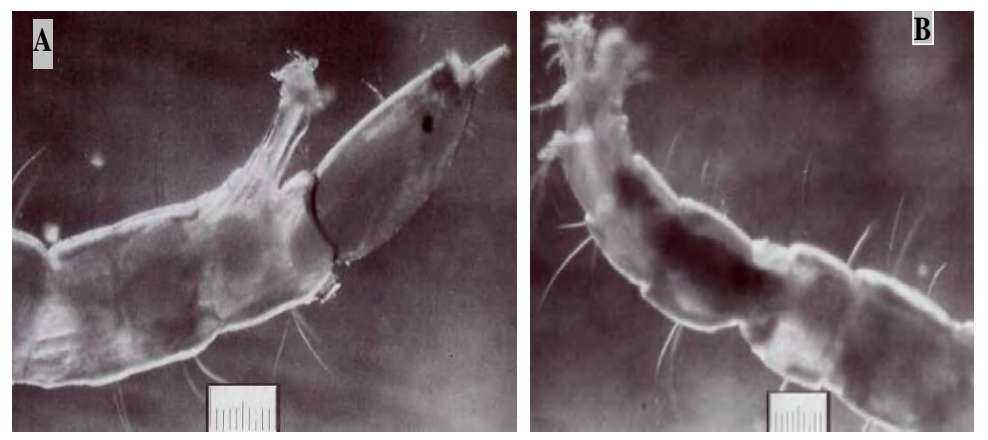

$50 \times 10$

Figure 5 : Micropelopiinae sp. (A = capsule céphalique ; $\mathrm{B}=$ soies sur le corps ; échelle sur la photo $=1 \mathrm{~mm})$. 
B. ALHOU et B. GODDEERIS / Int. J. Biol. Chem. Sci. 4(6): 2068-2081, 2010

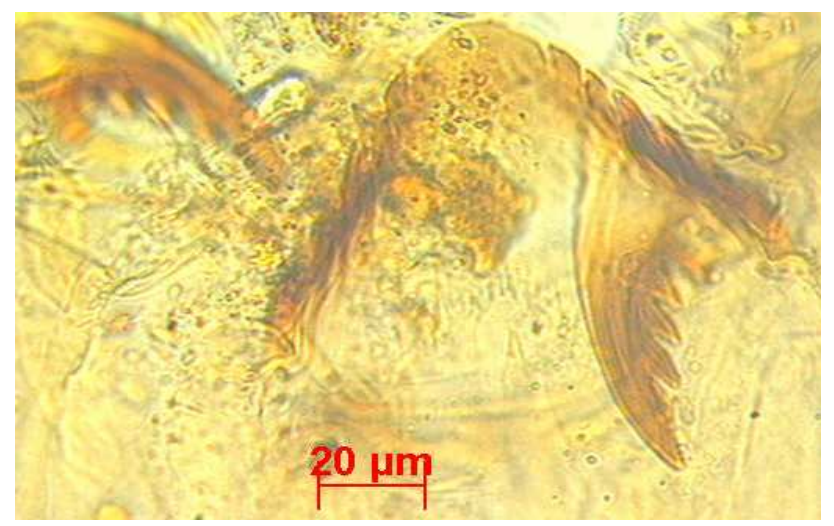

Figure 6 : Orthocladiinae sp. (mentum).
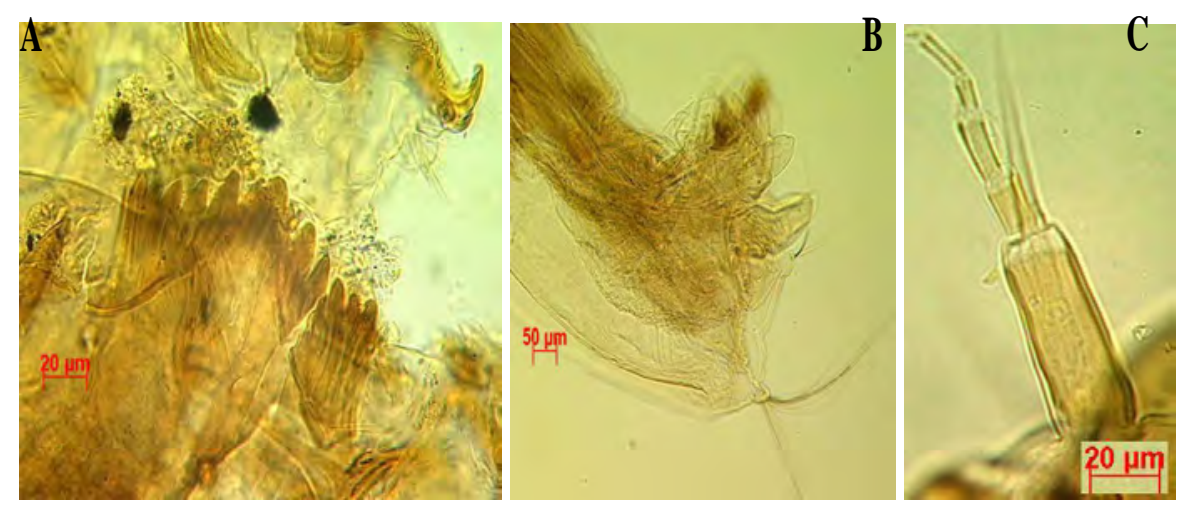

Figure 7 : Chironomini sp. 1 ( $\mathrm{A}=$ structure du mentum ; $\mathrm{B}=$ papilles anales $; \mathrm{C}=$ antenne $)$.
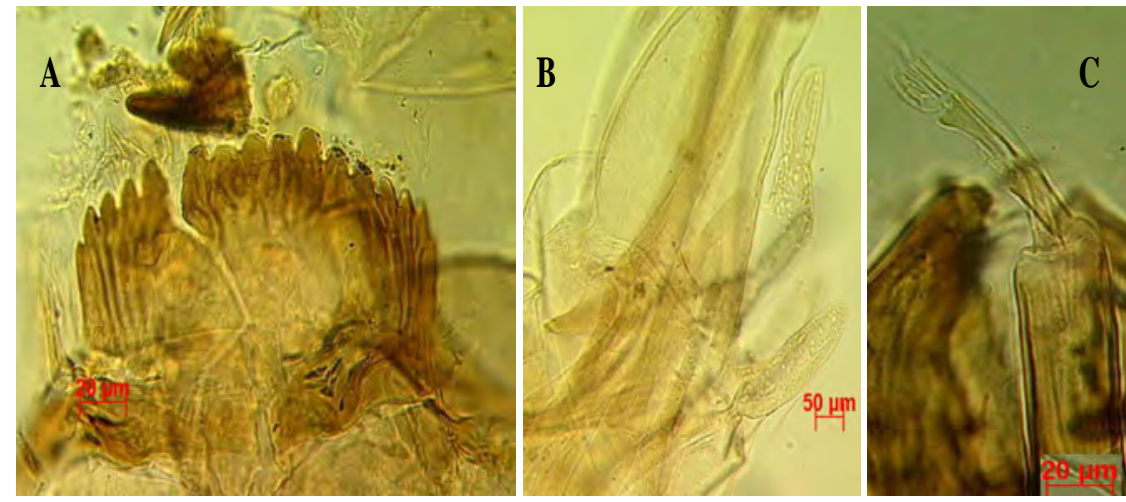

Figure 8 : Chironomini sp. 2 ( $\mathrm{A}=$ structure du mentum ; $\mathrm{B}=$ papilles anales ; $\mathrm{C}=$ antennes). 

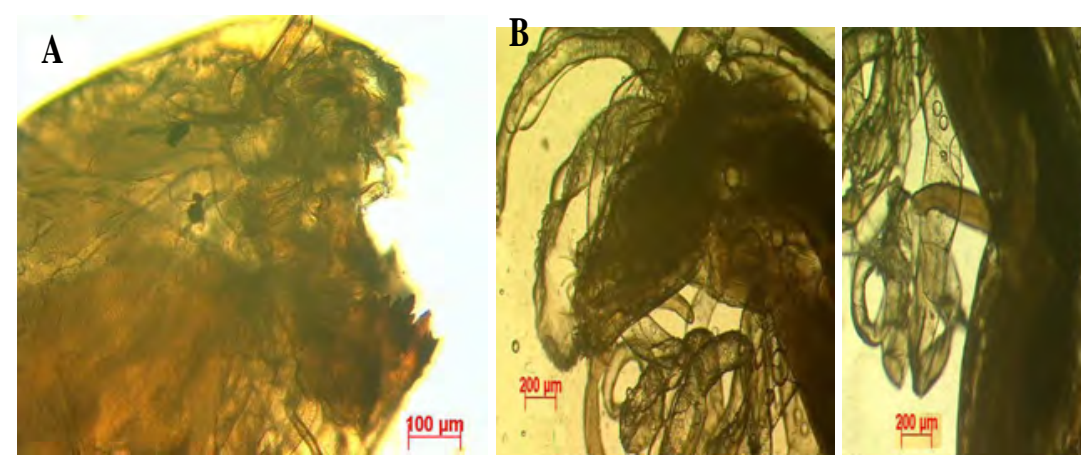

Figure 9 : Chironomus gr. plumosus $(\mathrm{A}=$ capsule céphalique $; \mathrm{B}=$ papilles anales $; \mathrm{C}=$ papilles anales et ventrales).

A
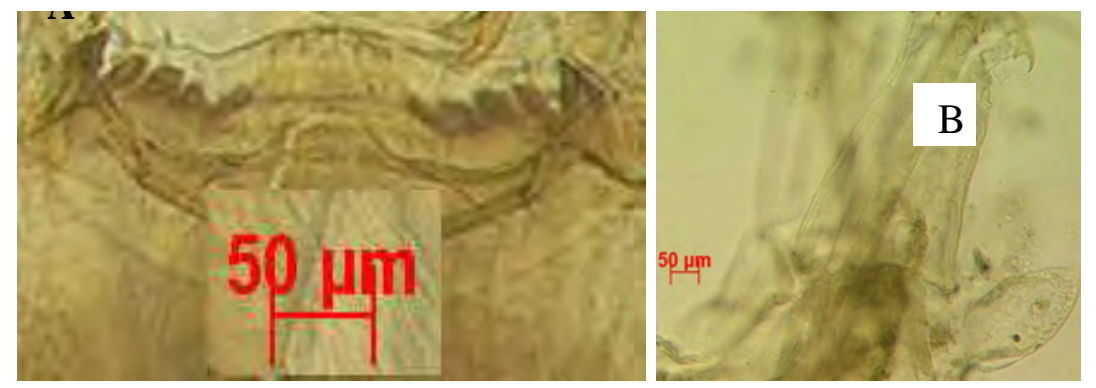

Figure 10 : Cryptochironomini $\mathrm{sp} .(\mathrm{A}=$ structure du mentum ; $\mathrm{B}=$ papilles anales).

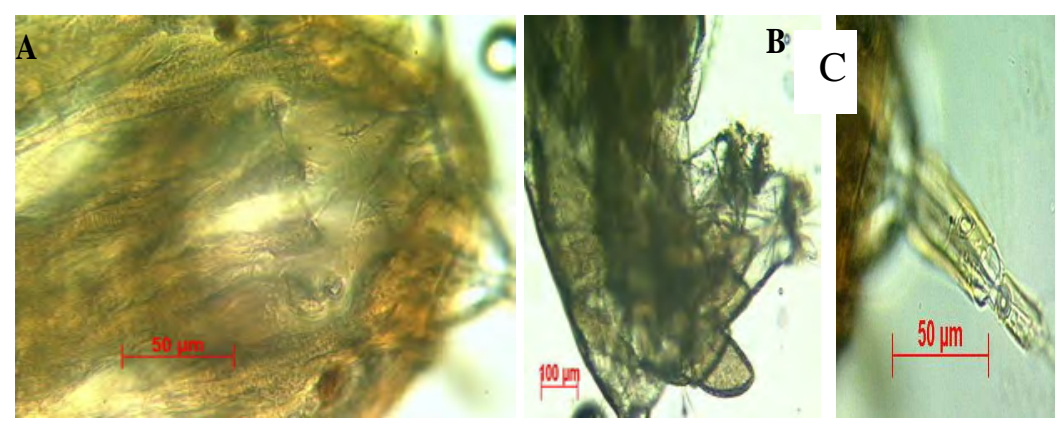

Figure 11: Cryptochironomus sp. $1(\mathrm{~A}=$ tâche blanche au mentum ; $\mathrm{B}=$ papilles anales ; $\mathrm{C}=$ antennes).
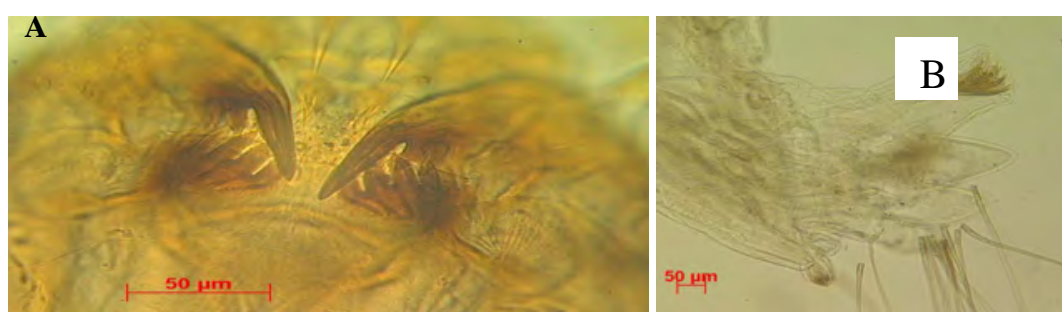

Figure 12 : Cryptochironomus sp.2 ( $\mathrm{A}=$ structure du mentum ; $\mathrm{B}=$ papilles anales). 2076 

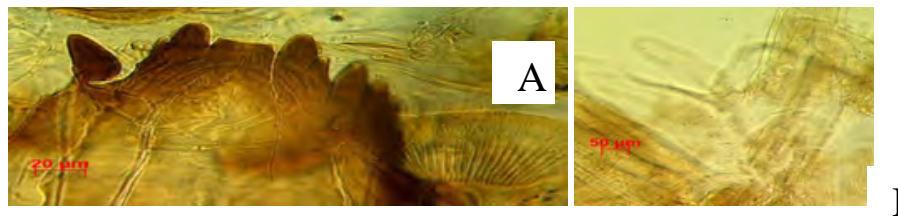

B
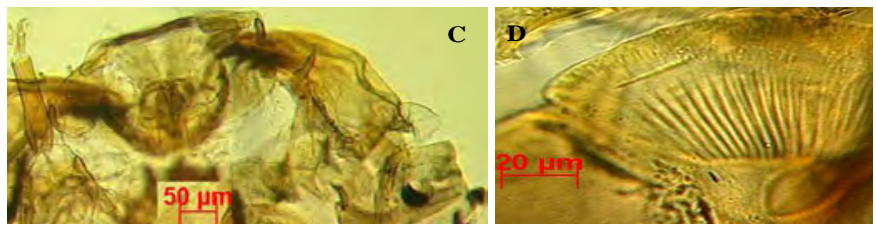

Figure 13 : Dicrotendipes sp. $(\mathrm{A}=$ structure du mentum $; \mathrm{B}=$ papilles anales $; \mathrm{C}=$ antenne $; \mathrm{D}=$ plaque paralabiale).
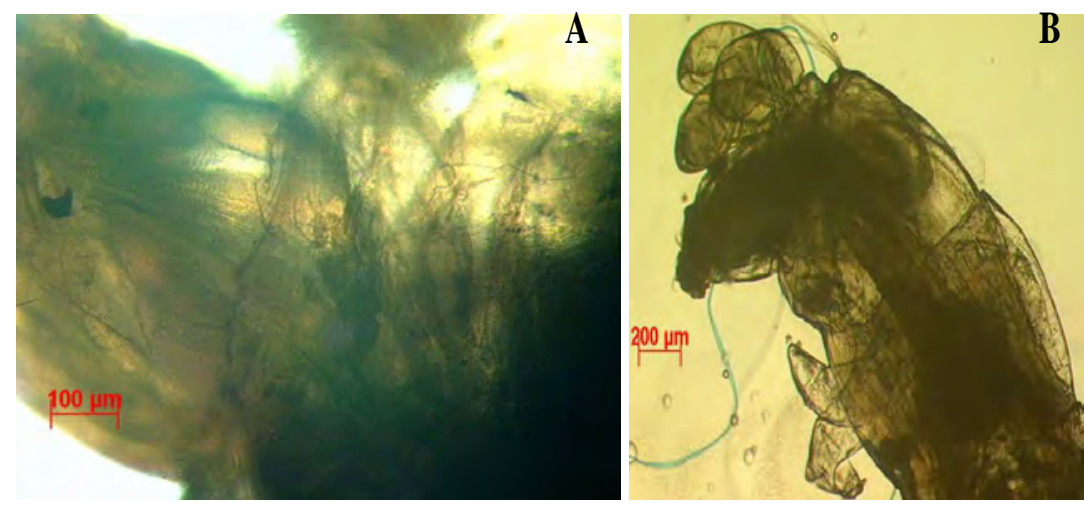

Figure 14 : Glyptotendipes sp. (A = enchevêtrement de la peau sur la capsule céphalique ; $\mathrm{B}=$ papilles anales et ventrales).
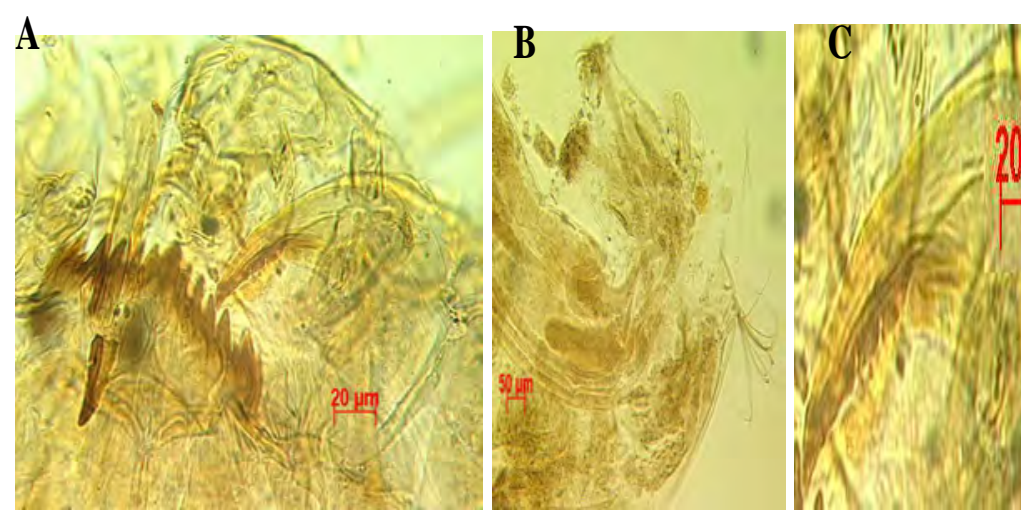

Figure 15 : Microchironomus sp. (A = structure du mentum ; $\mathrm{B}=$ papilles anales ; $\mathrm{C}=$ mandibules à dents carrées). 

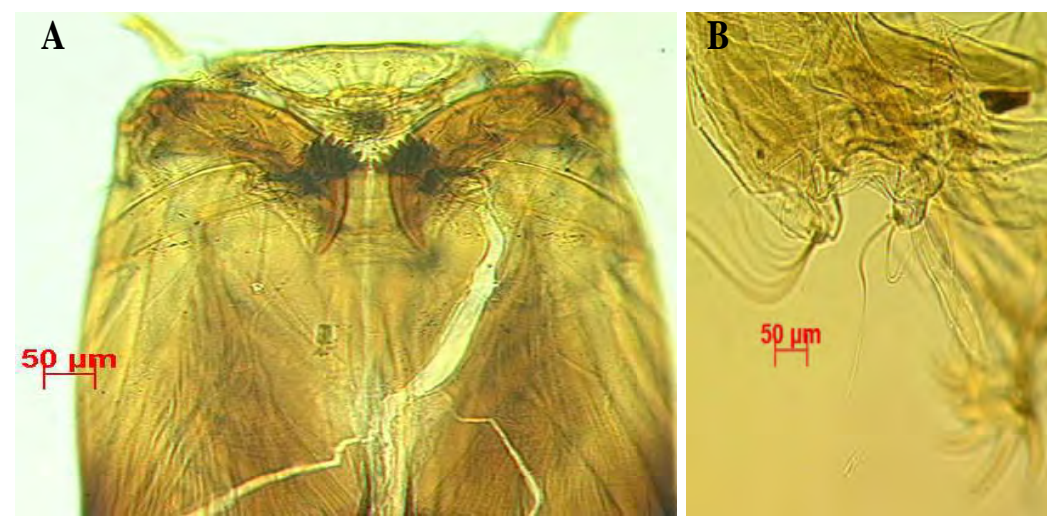

Figure 16 : Nilodosis $\mathrm{sp}$. (A = capsule céphalique avec mentum fortement coloré ; B = papilles anales).
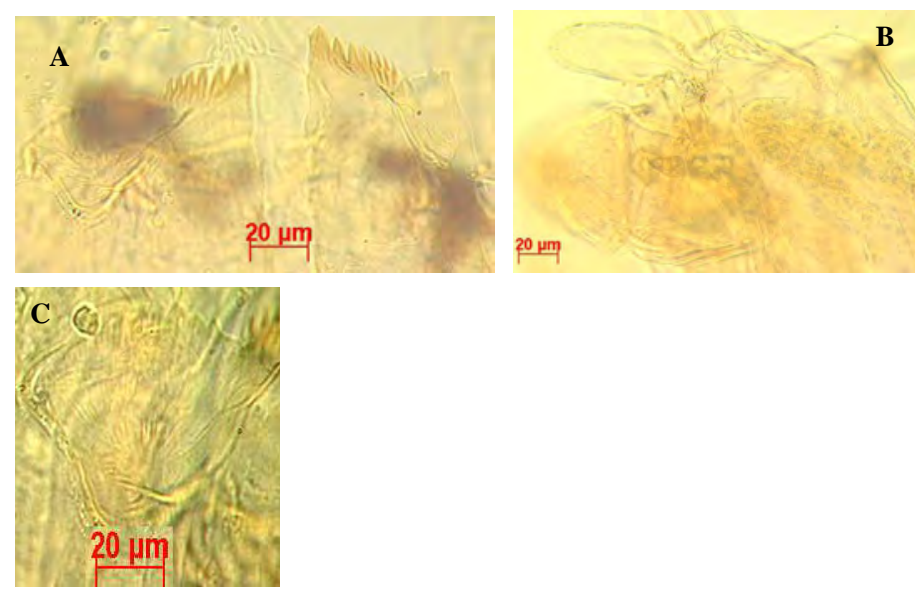

Figure 17 : Parachironomus sp. $(\mathrm{A}=$ structure du mentum $; \mathrm{B}=$ papilles anales $; \mathrm{C}=$ plaque paralabiale).

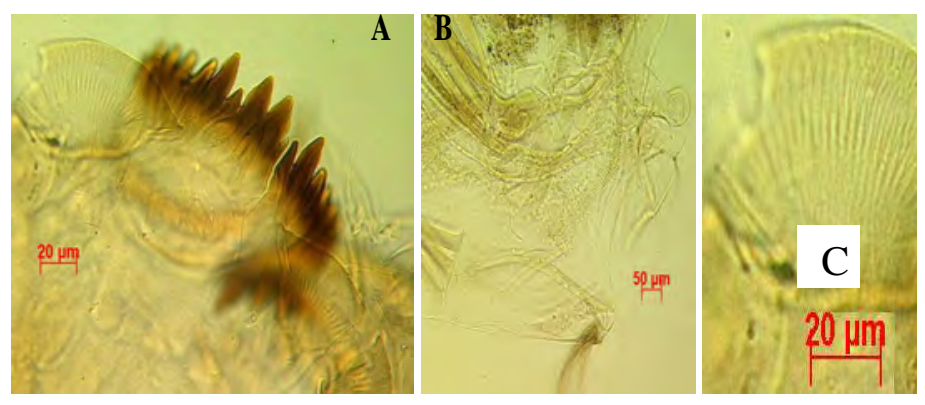

Figure 18: Polypedilum spp 1. ( $\mathrm{A}=$ structure du mentum $; \mathrm{B}=$ papilles anales $; \mathrm{C}=$ plaque paralabiale). 

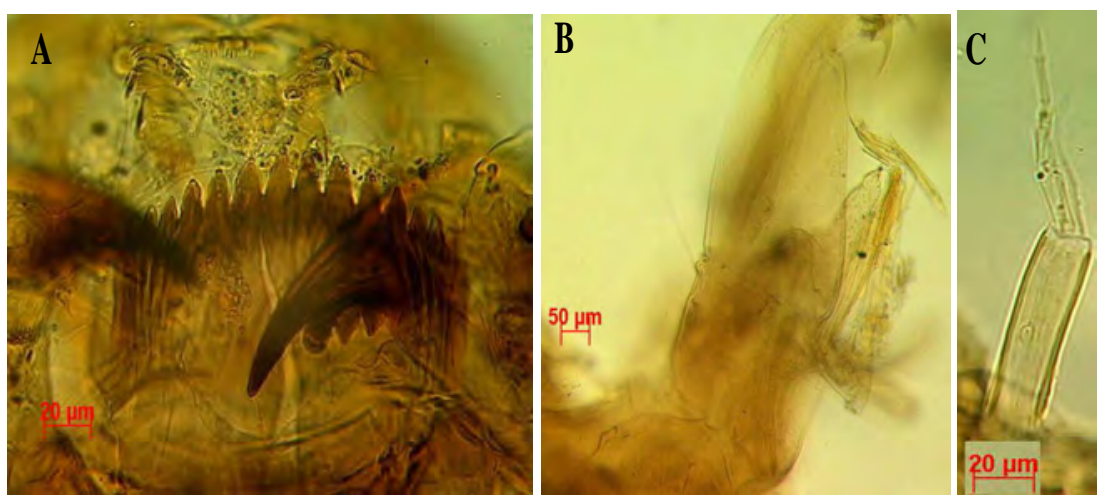

Figure 19 : Polypedilum spp. 2 ( $\mathrm{A}=$ structure du mentum ; $\mathrm{B}=$ papilles anales $; \mathrm{C}=$ antenne $)$.
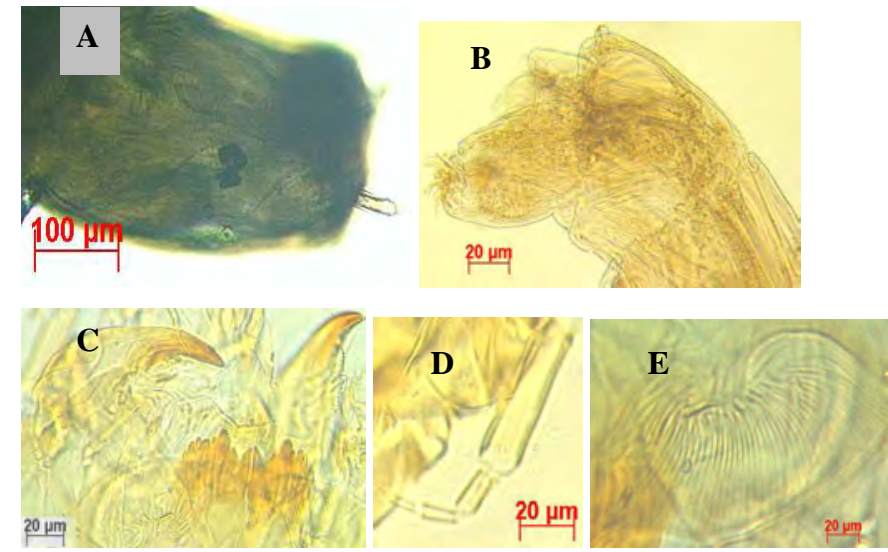

Figure 20 : Xenochironomus sp. ( $\mathrm{A}=$ capsule céphalique plus une paire des yeux ; $\mathrm{B}=$ papilles anales, $\mathrm{C}=$ mentum, $\mathrm{D}=$ antenne, $\mathrm{E}=$ plaque paralabiale $)$.

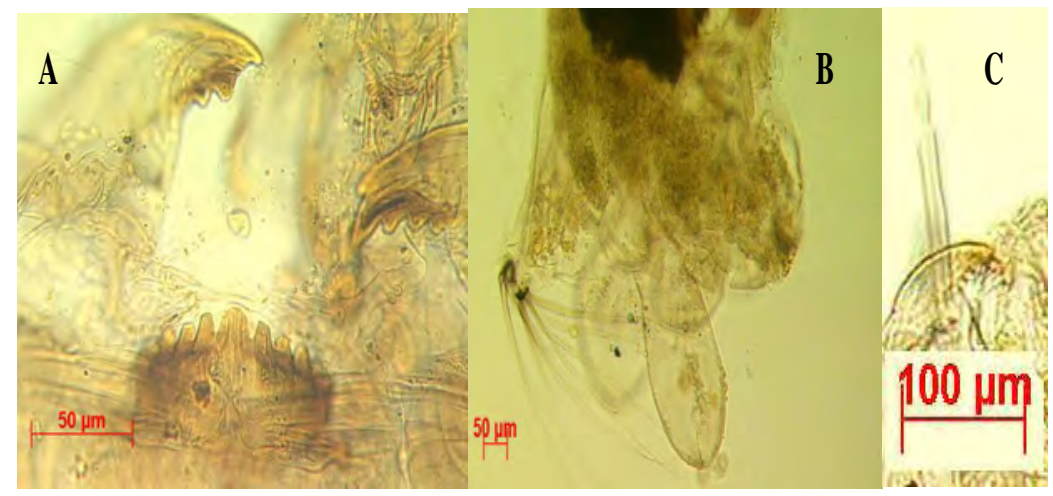

Figure 21 : Tanytarsini spp. $(\mathrm{A}=$ structure du mentum $; \mathrm{B}=$ papilles anales $; \mathrm{C}=$ antennes $)$. 


\section{DISCUSSION}

L'abondance et la diversité des Chironomidae de la zone d'étude auxquelles s'ajoutent leurs multiples avantages dans le management de la qualité de l'eau (Bauernfeind et Moog, 2000 ; Callisto et al., 2002 ; Hongqu et al., 2010 ) constituent un atout pour la mise au point d'un outil permettant de faciliter leur identification par les agents de terrain en charge du suivi de la qualité des eaux du fleuve Niger. La connaissance taxonomique des Chironomidae est alors indispensable pour leur utilisation comme bioindicateurs dans le management des ressources en eaux. Plusieurs travaux d'inventaire de la biodiversité chironomidienne en Afrique tropicale ainsi que les recherches taxonomiques s'inscrivent dans ce cadre (Kyerematen, 2000 ; Ekrem, 2001, 2003). Pour faciliter l' inventaire de la biodiversité chironomidienne du fleuve Niger à Niamey, cette étude propose une diagnose basée sur les caractéristiques morphologiques généralement bien visibles et assez utilisées dans l'identification des larves de Chironomidae (Eggermont et Verschuren, 2003 ; Eggermont et Verschuren, 2004a,b; Eggermont et al., 2005). Selon Dejoux (1984), le nombre d'espèces de Chironomidae en Afrique de l'Ouest s'élève à 96 au Togo et 31 au Niger. Ces chiffres dépassent ainsi de loin le nombre de spécimens présenté dans cette diagnose qui s'élève à 20 (Alhou, 2008). Ces spécimens sont repartis en 3 sous-familles à savoir les Chironominae, les Orthocladinae et les Tanypodinae avec respectivement 15,1 et 4 taxons. Ce nombre réduit de spécimen pourrait s'expliquer par le niveau taxonomique auquel cette diagnose se limite (Genre) et le champ d'étude qui comprend juste la portion du fleuve Niger dans la communauté urbaine de Niamey; ce qui n'est pas assez représentatif des types d'habitats que constituent les zones humides du Niger, lieux privilégiés pour le développement des larves de moustiques en général et des Chironomidae en particulier. Néanmoins, ces taxons sont assez représentatifs pour appréhender la qualité des eaux du fleuve Niger à Niamey (Alhou, 2009).

Cette première diagnose illustrée par des prises de vue est un premier pas dans l'identification des larves de Chironomidae qui aidera le personnel de terrain dans le premier trie des taxons et leur détermination au niveau générique. Ce travail devrait se poursuivre pour non seulement avoir un inventaire exhaustif des Chironomidae du fleuve Niger, mais aussi pour pousser cette diagnose jusqu'au niveau spécifique.

Bien qu'elle se limite au genre, cette diagnose constitue le premier inventaire de la biodiversité chironomidienne du fleuve Niger à Niamey et sert de base à une connaissance plus approfondie de cette famille des diptères assez répandue en Afrique tropicale (Diomandé et al., 2000 ; Vardal et al., 2002 ; Ole et Torbjørn, 2003). Elle représente ainsi un outil dans le management de la qualité des eaux de ce fleuve.

\section{REMERCIEMENTS}

Cette recherche a été financée par la Coopération Technique Belge (CTB), la Fondation Internationale pour la Science (FIS) et le Comité Scientifique et Technique de l'Organisation de la Conférence Islamique (COMSTECH), Islamabad, Pakistan.

\section{REFERENCES}

Bauernfeind E, Moog O. 2000. Mayflies (Insecta: Ephemeroptera) and the assessment of ecological integrity: a methodological approach. Hydrobiol., 422 (423) : 71-83.

Callisto M, Moreno P, Gonçalves JFJr, Leal JJF, Esteves FA. 2002. Diversity and biomass of Chironomidae (Diptera) larvae in an impacted coastal lagoon in Rio De Janeiro, Brazil. Braz. J. Biol., 62: 77-84.

Dejoux C. 1984. Contribution à la connaissance des Chironomides d'Afrique de l'Ouest (DiptèresNématocères), $3^{\mathrm{e}}$ note. Rev. Hydrobiol. Trop., 17: 65-76. 
Diomandé D, Gourène G, Sankaré Y, Zabi SG. 2000. Synopsis de la classification des larves et des nymphes de Diptères Chironomidae des écosystèmes dulçaquicoles de l'Afrique de l'Ouest. Clés de détermination des sous familles, des tribus et des genres. CRO, Abidjan. Arch. Sci., 17 (1): 1-31.

Eggermont H, Verschuren D. 2004a. Subfossil Chironomidae from East Africa. 1. Tanypodinae and Orthocladiinae. $J$. Paleolim., 32: 383-412.

Eggermont H, Verschuren D. 2004b. Subfossil Chironomidae from East Africa. 2. Chironominae (Chironomini and Tanytarsini). J. Paleolim., 32: 413-455.

Eggermont H, Verschuren D. 2003. Sub-fossil Chironomidae from Lake Tanganyika, East Africa. 1. Tanypodinae and Orthocladiinae. J. Paleolim., 29: 31-48.

Eggermont H, Verschuren D, Dumont $\mathrm{H}$. 2005. Taxonomic diversity and biogeography of Chironomidae (Insecta: Diptera) in lakes of tropical West Africa using subfossil remains extracted from surface sediments. J. Biogeogr., 32: 1063-1083.

Eggermont H. 2004. Fossil Chironomidae (Insecta, Diptera) as biological indicators for past salinity variation in African Lakes. Taxonomy, quantitative inference models, and assessment of model performance in space and time. Thèse de Doctorat, Univ. Gent, p.501.
Ekrem T. 2003. Towards a phylogeny of Tanytarsus van der Wulp. Is morphology alone sufficient to reconstruct the genealogical relationships between species in Chironomidae (Diptera). Insect Syst. Evol., 34: 199-219.

Ekrem T. 2001. A review of afrotropical Tanytarsus van der Wulp (Diptera: Chironomidae). Tijdschr. Entomol., 144: 5-40.

Hongqu T, Mi-Young S, Woon-Seok C, Young-Seuk P, Tae-Soo C. 2010. Species abundance distribution of benthic chironomids and other macroinvertebrates across different levels of pollution in streams. Ann. Lim. - Int. J. Lim., 46: 53-66.

Kyerematen RKA, Sæther O. 2000. A review of afrotropical Rheotanytarsus Thienemann et Bause (Diptera: Chironomidae). Tijdschr. Entomol., 143: 27-69.

Ole AS, Torbjørn E. 2003. Biogeography of afrotropical Chironomidae (Diptera), with special reference to Gondwanaland. Cimbebasia, 19: 123-139.

Vardal H, Bjørlo A, Sæther OA. 2002. Afrotropical Polypedilum subgenus Tripodura, with a review of the subgenus (Diptera: Chironomidae). Zool; Scripta, 31: 331-402. 